\title{
Identifying the Transgender Population in the Medicare Program
}

\author{
Kimberly Proctor, ${ }^{1,2, \dagger}$ Samuel C. Haffer, ${ }^{1, \dagger, *}$ Erin Ewald ${ }^{3}$ Carla Hodge, ${ }^{1}$ and Cara V. James ${ }^{1}$
}

\begin{abstract}
Purpose: To identify and describe the transgender population in the Medicare program using administrative data.

Methods: Using a combination of International Classification of Diseases ninth edition (ICD-9) codes relating to transsexualism and gender identity disorder, we analyzed 100\% of the 2013 Centers for Medicare \& Medicaid Services (CMS) Medicare Fee-For-Service (FFS) "final action" claims from both institutional and noninstitutional providers ( $\sim 1$ billion claims) to identify individuals who may be transgender Medicare beneficiaries. To confirm, we developed and applied a multistage validation process.

Results: Four thousand ninety-eight transgender beneficiaries were identified, of which 90\% had confirmatory diagnoses, billing codes, or evidence of a hormone prescription. In general, the racial, ethnic, and geographic distribution of the Medicare transgender population tends to reflect the broader Medicare population. However, age, original entitlement status, and disease burden of the transgender population appear substantially different. Conclusions: Using a variety of claims information, ranging from claims history to additional diagnoses, billing modifiers, and hormone prescriptions, we demonstrate that administrative data provide a valuable resource for identifying a lower bound of the Medicare transgender population. In addition, we provide a baseline description of the diversity and disease burden of the population and a framework for future research.
\end{abstract}

Keywords: administrative data; disease burden; intersectionality; Medicare; transgender

\section{Introduction}

Despite increased awareness and greater societal acceptance of people who are transgender, the inability to systematically identify and study the transgender population greatly hampers our capacity to conduct meaningful analysis of this group. Minimal representative national data exist, ${ }^{1}$ studies attempting to estimate the size and health needs of the transgender population have generally relied on nonprobability survey samples, ${ }^{2}$ and analyses utilizing more robust research designs have largely focused on single states. ${ }^{3}$ Furthermore, population-based studies of transgender individuals entitled to Medicare due to age (65 and older), disability, or end-stage renal disease are nonexistent, demonstrating the need for more and better research focused on sexual and gender minorities, including the transgender population. Toward this end, recent research conducted at the Department of Veterans Affairs suggests the potential utility of using healthcare administrative data to identify persons who are transgender. ${ }^{4}$ Expanding on this work, we explore the use of Medicare billing data from the Centers for Medicare \& Medicaid Services (CMS), the federal agency that administers the Medicare and Medicaid programs, to identify and describe Medicare's transgender population.

\footnotetext{
${ }^{1}$ Office of Minority Health, U.S. Centers for Medicare \& Medicaid Services, Baltimore, Maryland.

${ }^{2}$ Center for Medicaid and CHIP Services, U.S. Centers for Medicare \& Medicaid Services, Baltimore, Maryland.

${ }^{3}$ NORC at the University of Chicago, Chicago, Illinois and Bethesda, Maryland.

${ }^{\dagger}$ Cofirst authors.
}

*Address correspondence to: Samuel C. Haffer, PhD, Office of Minority Health, U.S. Centers for Medicare \& Medicaid Services, 7500 Security Boulevard [MS: S2-12-17], Baltimore, MD 21244-1850, E-mail: chris.haffer@cms.hhs.gov

U.S. Centers for Medicare \& Medicaid Services. 2016; Published by Mary Ann Liebert, Inc. This Open Access article is in the public domain and may be used and reprinted without permission. 
The transgender population includes individuals whose gender identity, gender expression, or gender behavior does not typically conform to the sex they were assigned at birth. ${ }^{5}$ This community experiences a particularly high disease burden, including significantly higher rates of substance abuse, ${ }^{6-8}$ HIV/AIDS, ${ }^{9-11}$ and mental illness. ${ }^{10,12,13}$ Discrimination in the healthcare setting only exacerbates these adverse health outcomes. Twenty-eight percent of transgender persons report postponing medical care when sick due to discrimination, $19 \%$ report that doctors have refused to provide them care because of their transgender status, $28 \%$ report facing harassment in the medical setting, $2 \%$ report facing violence in a doctor's office, and $>50 \%$ report that they had to teach their doctor about transgender healthcare. ${ }^{2}$ Taken together, transgender persons experience suboptimal health outcomes across a variety of areas while systematically lacking access to the institutions that have the ability to address these medical needs.

Even when transgender persons are able to receive care, insurers routinely deny treatment related to medical transitions. Transitioning is the process of living as the gender with which a transgender person identifies, rather than the gender assigned to them at birth. ${ }^{2}$ Medical transitions include any type of transgender-related surgery, such as sex-reassignment surgery or cosmetic procedures, and hormone therapy, such as taking prescriptions for cross-sex hormones. Medical transitions are particularly relevant for the Medicare program, which covers certain aspects of these medical treatments and includes this information in Medicare claims data.

Until 2015, providers treating patients enrolled in Medicare used the International Classification of Diseases ninth edition (ICD-9) to indicate a patient's specific medical diagnoses when submitting medical claims to CMS. ICD-9 contains multiple diagnosis codes that are transgender specific, including the following codes ${ }^{14}$ : 302.50 (Transsexualism with unspecified sexual history), 302.51 (Transsexualism with asexual history), 302.52 (Transsexualism with homosexual history), 302.53 (Transsexualism with heterosexual history), 302.6 (Gender Identity Disorder [GID] in children), and 302.85 (GID in adolescents or adults).

CMS also advises providers to utilize two billing modifiers that apply to the transgender population, including the condition code 45 modifier and the KX modifier. Medicare billing modifiers are two-digit codes appended to procedure codes or Healthcare Common Procedure
Coding System (HCPCS) codes that provide additional information about the billed procedures. ${ }^{15,16}$ Providers use billing modifiers to avoid rejection of claims with a gender/procedure conflict. For example, the CMS system will reject a claim where a physician provided a female pelvic examination for a male beneficiary, as female pelvic examinations are considered sex specific (i.e., only for females). Because transgender beneficiaries may have changed their sex on record, they are at a high risk for experiencing gender/procedure conflicts. In this instance, a transman (female to male transition) may have his claim for a medically necessary pelvic examination rejected inappropriately. Therefore, condition code 45 and the KX modifier are used to process claims with gender-specific editing that CMS would normally reject due to gender/procedure mismatches. A list of the gender-specific procedure codes related to condition code 45 and the KX modifier is included in Appendix Table 1.

Similar to diagnosis codes and billing modifiers, CMS also maintains a record of each Medicare beneficiary's prescriptions. The Medicare Part D prescription drug plan covers medically necessary hormones for transgender persons, such as cross-sex hormones. Records of these prescriptions are available in CMS's administrative files and include the generic and brand names of prescription drugs, as well as details about the prescription. A list of hormone therapy-related prescription drugs is included in Appendix Table 2.

As a result, it may be possible to identify transgender Medicare beneficiaries using one or a combination of these diagnosis codes, billing modifiers, and prescription drug events.

\section{Methods}

Utilizing the CMS Chronic Conditions Data Warehouse (CCW), which contains CMS data on Medicare and Medicaid beneficiaries and their claims, we analyzed $100 \%$ of the CMS Fee-For-Service (FFS) final action claims from both institutional and noninstitutional providers for calendar year 2013. These claims included inpatient and outpatient hospital claims, carrier claims (e.g., physicians, physician assistants, nurse practitioners), and claims from skilled nursing facilities, home health agencies, hospice care, and those relating to durable medical equipment. In total, this covered $\sim 1$ billion claims.

In the first component of the analysis, we searched each claim for any occurrence in any position of diagnosis codes $302.50,302.51,302.52,302.53,302.6$, or 
302.85. Once we identified the universe of claims meeting our criteria, we used the unique Medicare beneficiary identifier present on each claim to identify unique observations. Following this identification process, we used the unique beneficiary identifier to link to the Medicare Enrollment and Medicare Part D Data in the CCW.

Because administrative records contain a degree of error and the billing modifiers are not unique to the transgender community, the data potentially contain a high probability of producing false positives, in which nontransgender beneficiaries are identified as transgender. To address this concern, we developed a supplementary method for validating the initial classification. The first validation step analyzes the repeated application of ICD-9 codes 302.50, 302.51, 302.52, 302.53 , 302.6, and/or 302.85, with persons receiving more than one diagnosis in 2013 having a validated classification. The second and third validation steps analyze the relevant ICD-9 codes over time. If the beneficiary had one or more of these diagnoses in the preceding year (2012) or subsequent year (2014), indicating an ongoing trend of receiving the diagnosis, the classification was validated. The fourth validation step incorporated data on ICD-9 code 259.9 (Unspecified Endocrine Disorder), which is frequently used by the transgender community to combat the perceived stigma of a GID diagnosis. If a beneficiary received at least one diagnosis from the transgender-specific ICD-9 codes and also received a diagnosis of 259.9, the classification was validated. The fifth validation step incorporated prescriptions for sex hormones, with persons receiving a transgender-specific diagnosis code and a prescription for a sex hormone representing a validated classification. The sixth validation step examined the principal diagnosis code and, if the principal diagnosis code was from a transgender-specific ICD-9 code, that observation was validated. Finally, the seventh and eighth validation steps incorporated the billing claims modifiers to validate classifications. If a beneficiary received a relevant ICD-9 code and had at least one claim containing the condition code 45 modifier or the KX modifier, the classification was validated.

Given the limitations of using ICD-9 259.9, sex hormones, and claim modifiers to identify transgender Medicare beneficiaries, these aspects of medically transitioning were only included as validation steps, rather than unique identifiers. Although this conservative approach restricts the size of the cohort, it is the only mechanism for guaranteeing that nontransgender per- sons are not misclassified as transgender. To demonstrate, there were over 5000 Medicare beneficiaries in 2013 with a claim containing the KX modifier or condition code 45 and a gender/procedure conflict, with only $3.90 \%$ of these beneficiaries receiving a transgender-specific ICD-9 diagnosis code. Given our limited ability to determine if the remaining $96.10 \%$ of these beneficiaries are transgender or not, we recommend that researchers avoid utilizing these modifiers alone and incorporate additional data, such as ICD-9 codes, to classify beneficiaries as transgender.

\section{Results}

Enumerating Medicare's transgender population

Using this methodology, we identified 4098 persons as transgender Medicare beneficiaries. Table 1 demonstrates these findings along with results from the validation logic. This classification method was highly accurate, with $89.26 \%$, or 3658 persons, having enough information in their claims history to validate their classification as transgender. This demonstrates that researchers interested in studying Medicare's transgender population can identify a meaningfully large and accurate population using ICD-9 codes in conjunction with supplementary claims data. This does not imply that the $10.74 \%$ of observations not validated are incorrectly classified or that this method identifies all transgender persons enrolled in Medicare, rather, it provides a conservative estimate (lower bound) of Medicare's transgender population and details a methodology for identifying and validating this population using administrative data. Consequently, these tools provide a replicable foundation for researchers interested in analyzing health outcomes in the transgender community.

Table 1 also demonstrates the validation results in greater detail. For individuals identified using only ICD-9 codes, the majority of beneficiaries $(66.03 \%)$ had more than one claim with a transgender-specific ICD-9 code within the calendar year. Other validation methods, such as using claims from bordering calendar years and hormone prescriptions, had very similar results. Approximately, forty percent of the beneficiaries identified by transgender-specific ICD-9 codes had similar claims in 2012, 2014, filled a prescription for a sex hormone in 2013, or received a transgender-specific principal diagnosis code. A considerably smaller number of transgender beneficiaries had claims with ICD-9 code 259.9 or billing modifiers, although these validation methods did validate $>700$ observations. In total, the results indicate that our validation methodology 
Table 1. Identification and Validation Logic

\begin{tabular}{|c|c|c|c|}
\hline \multicolumn{4}{|l|}{ Transgender Medicare Beneficiaries } \\
\hline & No. identified & No. validated & $\%$ validated \\
\hline \multirow{2}{*}{$\begin{array}{l}\text { ICD-9 diagnosis codes } 302.50,302.51,302.52,302.53,302.6,302.85^{\text {a }} \\
\text { Validation method } \\
\text { ICD-9 } 302 \text { series diagnosis code and } 1 \text { or more of the following: }\end{array}$} & 4098 & 3658 & 89.26 \\
\hline & & No. validated & $\%$ validated \\
\hline More than 1 claim with an ICD-9 302 series diagnosis code in 2013 & & 2706 & 66.03 \\
\hline 1 or more claims with an ICD-9 302 series diagnosis code in 2012 & & 1577 & 38.48 \\
\hline 1 or more claims with an ICD-9 302 series diagnosis code in 2014 & & 1937 & 47.26 \\
\hline 1 or more claims with an ICD-9 259.9 diagnosis code in 2013 & & 568 & 13.86 \\
\hline 1 or more prescriptions for a sex hormone in 2013 & & 2005 & 48.89 \\
\hline Principal diagnosis code is from ICD-9 302 series & & 1736 & 42.36 \\
\hline 1 or more CC 45 modifier & & 167 & 4.08 \\
\hline 1 or more $\mathrm{KX}$ modifier & & 26 & 0.6 \\
\hline
\end{tabular}

Each validation step is calculated independently from all other validation steps, and "\% validated" is calculated from the total number of transgender beneficiaries identified $(N=4098)$.

${ }^{\text {a }}$ Referred to as the 302 series for the purposes of this table.

ICD-9, International Classification of Diseases ninth edition.

supplements the initial classifications by incorporating additional detail and analyzing the validity of using administrative data to identify the transgender population.

\section{Demographic variability in Medicare's} transgender population

Using this foundation to identify transgender Medicare beneficiaries, analyzing their demographic characteristics also helps describe this population. Results demonstrate that Medicare's transgender population is racially and ethnically diverse, spans the entire United States, and experiences many chronic conditions. Analyses reported here utilize the entire cohort of 4098 individuals identified as transgender (3658 identified and validated through administrative data and 440 identified but not validated through administrative data). We conducted separate analyses (not shown), which excluded the 440 individuals for whom we have no additional claims-based validation information. However, there were no systematic or substantive differences in the results. Therefore, we report results on the entire cohort.

Beginning with race, the data demonstrate that the transgender Medicare population contains members from all racial and ethnic groups. This population is racially and ethnically diverse, with substantial representation among Whites, Blacks/African Americans, and Hispanics. Figure 1 displays the distribution of racial

"Due to the high degree of error in Centers for Medicare \& Medicaid Services (CMS's) race/ethnicity data this analysis uses CMS's RTI race code to identify a beneficiary's race. ${ }^{17-19}$ and ethnic identity within the transgender population. In this population of transgender persons, Whites comprise $73.99 \%$ of the total population, Blacks/African Americans comprise the next largest group, representing $15.37 \%$ of the transgender Medicare population, and Hispanics, Asians/Pacific Islanders (APIs), American Indians/Alaska Natives (AIANs), Unknowns, and Others comprise relatively smaller proportions of the transgender Medicare population. This analysis of the racial and ethnic diversity of the transgender population is significant, as $>85 \%$ of studies that examine sexual and gender minorities fail to report data on race. $^{20}$ This lack of data on the racial and ethnic diversity of transgender persons inhibits our ability to understand the intersectionality of gender identity and racial/ethnic identity, which is expected to have important effects on health outcomes. Because research has consistently identified the prevalence of minority health disparities, ${ }^{21-26}$ these disparities may disproportionately affect the diverse transgender community. Therefore, understanding how race and ethnicity interact with transgender identity is an important component of studying transgender health and this analysis provides the foundation for future research on this topic.

The transgender population enrolled in the Medicare program displays a high level of geographic diversity. Figure 2 demonstrates that transgender Medicare beneficiaries reside in every state, with many states containing large populations. California contains the largest number of transgender Medicare beneficiaries, 


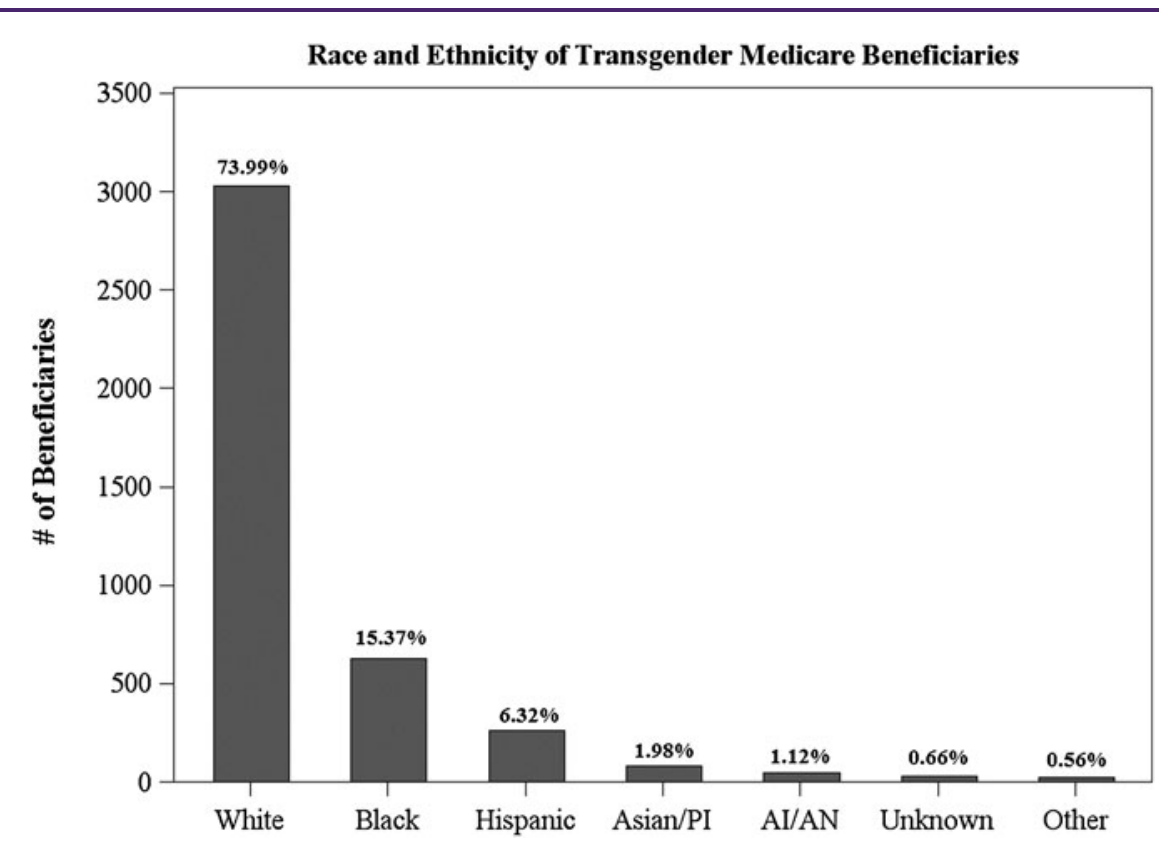

FIG. 1. Racial/ethnic identification of transgender beneficiaries.

with 562 beneficiaries. New York (282), Texas (201), with each state containing 100 or more transgender Florida (198), Massachusetts (179), Washington beneficiaries. This is an important finding, as it demon(173), Ohio (146), Minnesota (146), Michigan (145), strates that the transgender population spans the entire Pennsylvania (116), Illinois (115), Wisconsin (101), United States, making transgender health relevant to and Georgia (100) also contain large populations, local providers across the entire country.

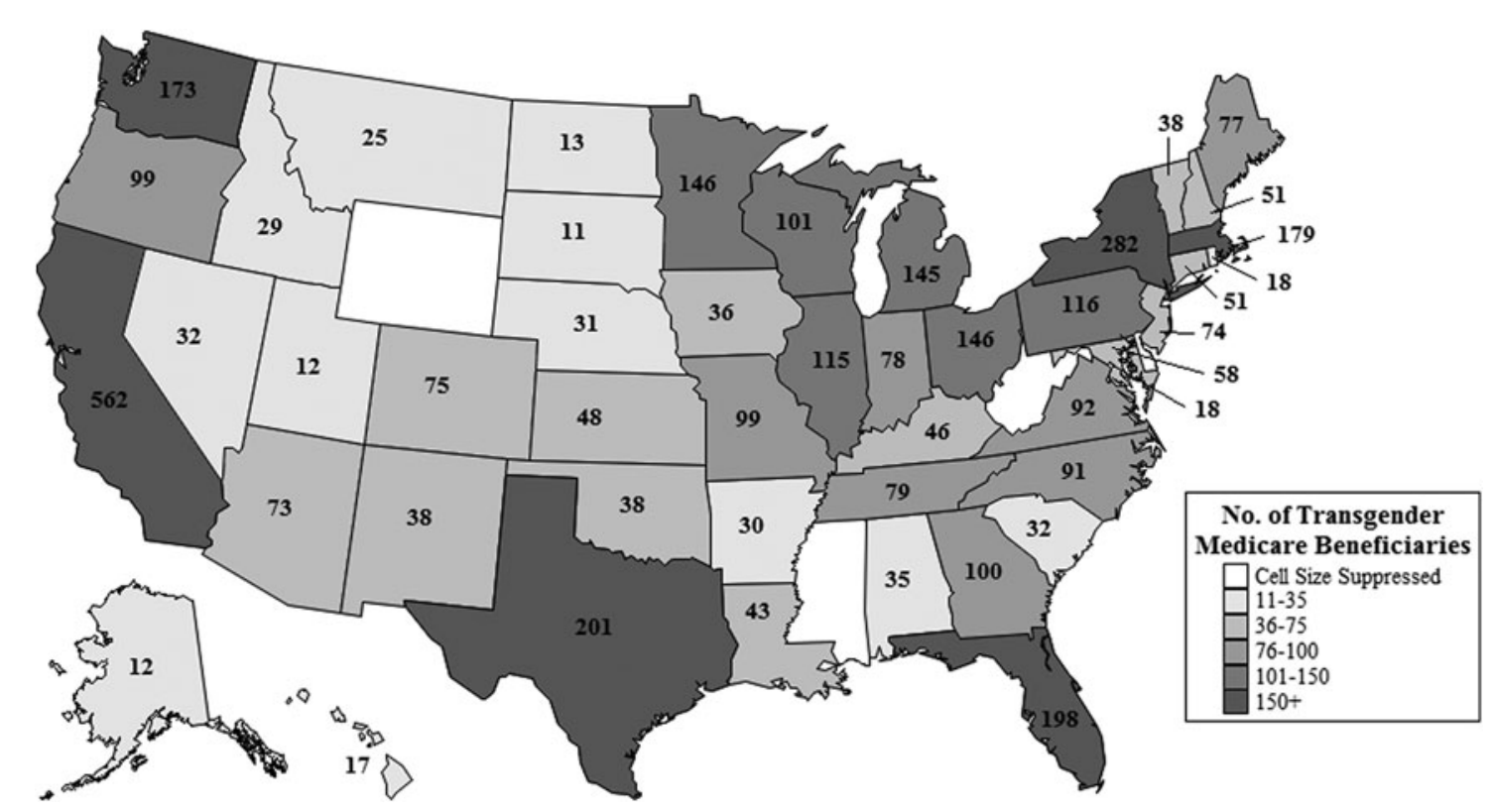

FIG. 2. Geographic distribution of transgender beneficiaries. 


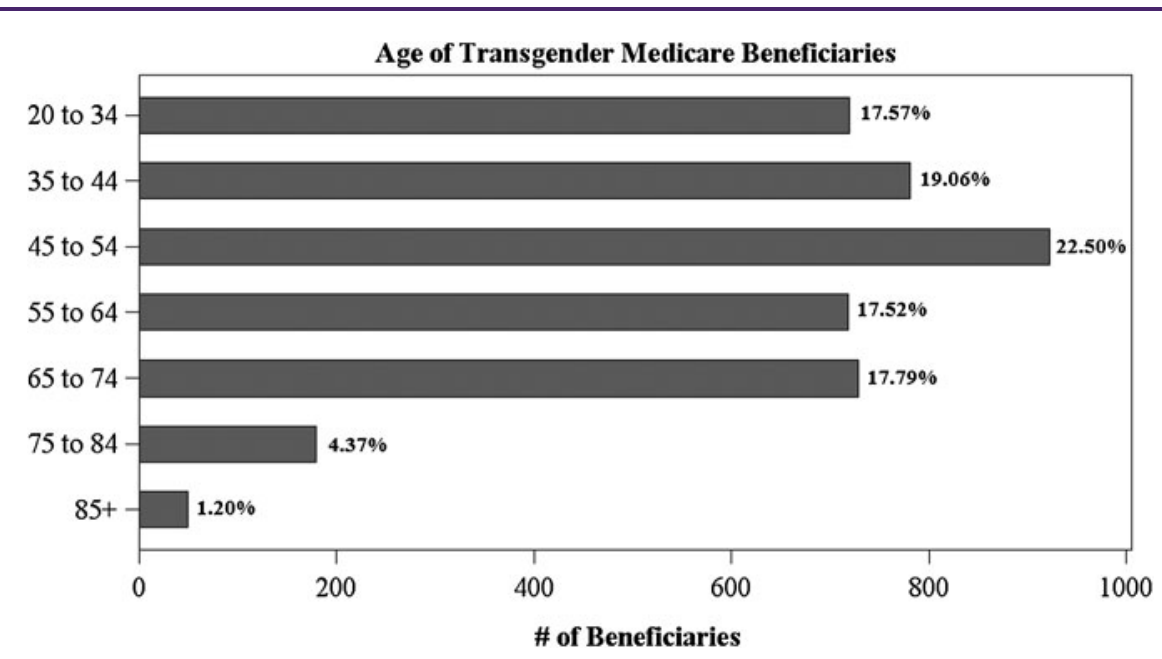

FIG. 3. Age distribution of transgender beneficiaries.

Unlike the racial, ethnic, and geographic distribution of the transgender population, which tends to reflect broader population distributions, the age, original entitlement status, and chronic condition burden of the transgender population appear substantially different. Figure 3 displays the age distribution of the transgender population, showing that the majority of transgender Medicare beneficiaries were under age 65 in 2013 (76.65\%). This is a somewhat surprising result, as age is the primary mechanism through which most Americans qualify for Medicare. To demonstrate, $75.55 \%$ of the general Medicare population qualified for Medicare through Old Age and Survivors Insurance (OASI), indicating that the majority are age 65 or older. The transgender Medicare population, conversely, primarily qualified for Medicare through Disability Insurance (84.06\%), implying that many transgender persons enrolled in the program are disabled. This trend reflects an almost exact reversal of the general population's Medicare eligibility. Thus, the transgender population may be disproportionately disabled relative to the general Medicare population, which suggests an avenue for future research that examines these differences.

Using CMS's chronic condition categories, which analyze 60 chronic medical conditions and other chronic or potentially disabling conditions, Figure 4 highlights the chronic condition prevalence in the Medicare transgender population, demonstrating the significant burden placed on many beneficiaries. This is particularly relevant for depression, which has affected $81.79 \%$ of those under the age of 65 . Because three-quarters of the transgender population has been diagnosed with depression at some point during their life, the data suggest that the community disproportionately suffers from depression. Other mental health issues, such as posttraumatic stress disorder, schizophrenia, psychotic disorders, anxiety disorders, and major depressive affective disorders, also affect a large proportion of the population, demonstrating the significant mental health burden facing transgender Medicare enrollees. This echoes findings from previous studies, ${ }^{13}$ which report that there is a high prevalence of depression in the transgender community and transgender persons are more likely to report depression if they have not begun a medical transition. This finding suggests an opportunity for future research that examines the role that receiving medically necessary treatment may play in reducing depression rates and improving the mental health of transgender beneficiaries.

Hyperlipidemia and hypertension also affect the majority of transgender beneficiaries, with $58.49 \%$ of beneficiaries reporting either condition. This is especially relevant for those under the age of 65 , with a majority of those in this age category reporting these chronic conditions, even though they are typically associated with advancing age. ${ }^{26,27}$ This is consistent with previous studies on the transgender population ${ }^{28}$ and suggests a need for additional research that analyzes the association between medical transitions and hyperlipidemia/hypertension, which appear to affect a statistically high proportion of the transgender population, relative to their age. Other conditions, such as tobacco 


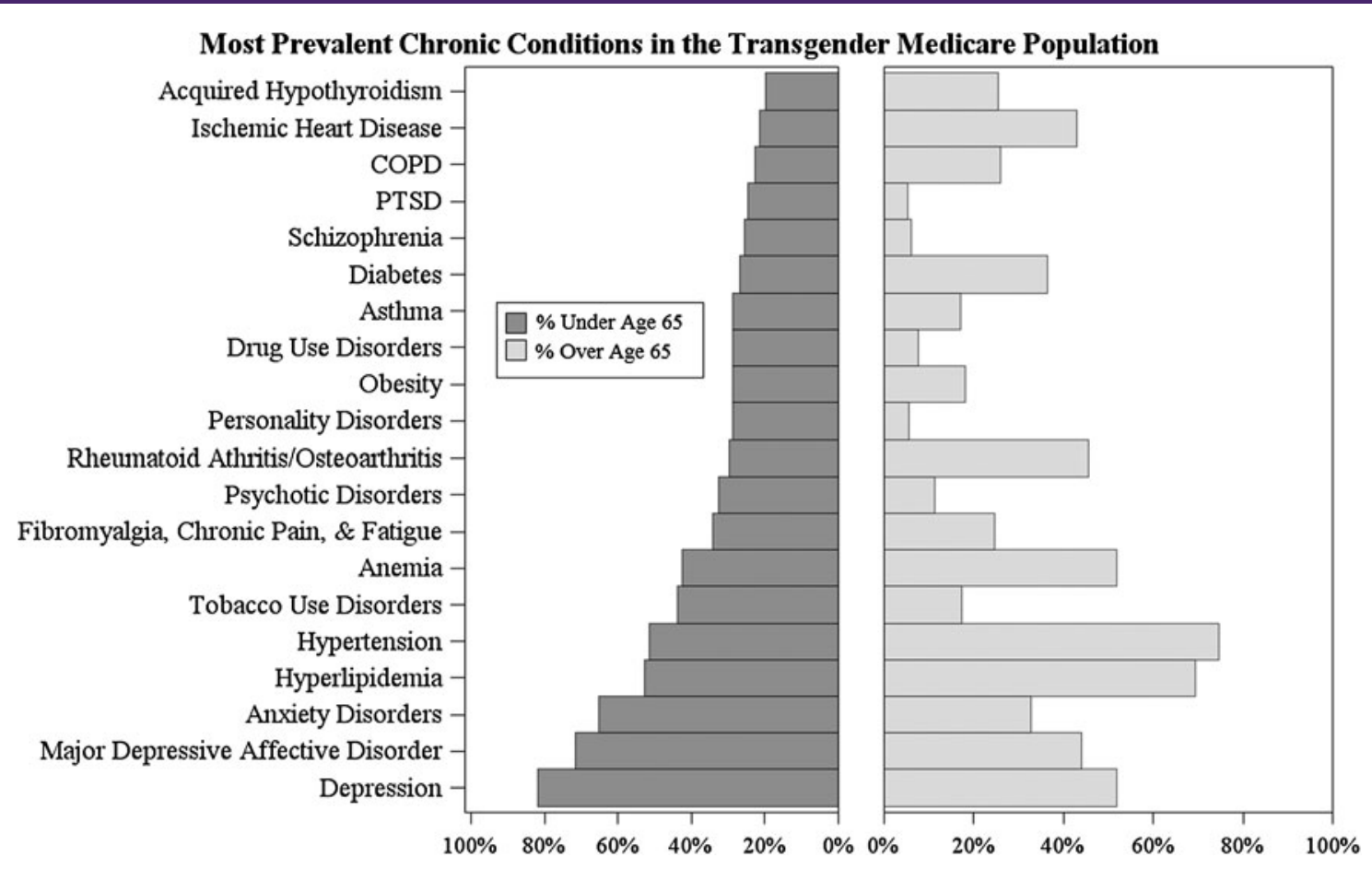

FIG. 4. Chronic conditions and the transgender Medicare population.

and drug use disorders, fibromyalgia, and other forms of chronic pain or fatigue, obesity, anemia, rheumatoid arthritis/osteoarthritis, asthma, diabetes, and heart disease, affect more than one-quarter of the population and reflect the broader trend of the transgender community reporting a disproportionately high disease burden. Future research that examines the causes of these high prevalence rates would help inform the treatment of transgender Medicare beneficiaries and explain why these diseases are manifesting in transgender persons at early ages.

\section{Discussion}

Using CMS's administrative data, we were able to identify and validate nearly 3700 transgender beneficiaries enrolled in Medicare during the 2013 calendar year. Using a variety of claims information, ranging from claims history to additional diagnoses, billing modifiers, and hormone prescriptions, we demonstrate that administrative data provide a valuable resource for studying the transgender population. ICD-9 codes specific to medical transitions are especially useful, with $90 \%$ of those identified using this method being validated. Therefore, ICD-9 codes provide an excellent foundation for future research on the transgender pop- ulation, and we encourage researchers interested in transgender health and health outcomes to utilize this methodology for future research.

The resulting cohort of transgender Medicare beneficiaries also demonstrates the significant racial, ethnic, and geographic diversity of the population. The results indicate that the transgender population is very diverse, containing members of every racial and ethnic group and residing in every U.S. state. Because fewer than $15 \%$ of studies on the health status of lesbian, gay, bisexual, and transgender (LGBT) persons include an analysis of race, ${ }^{20}$ this examination provides an important contribution to health services research. The geographic distribution of transgender Medicare beneficiaries also provides important implications for transgender-specific care. Given that $>50 \%$ of transgender persons report having to teach their provider about transgender healthcare, ${ }^{2}$ these results suggest that providers across the nation should better prepare for providing care to Medicare's transgender population, as there is a high probability that providers may encounter transgender patients. This is particularly relevant, given the lack of LGBT outreach across the country, with few agencies providing LGBT-specific training or outreach. ${ }^{29}$ Because agencies that provide 
LGBT-specific services are more likely to address LGBT issues, receive LGBT assistance requests, and understand the unique needs facing the community, ${ }^{29}$ these results provide support for increasing education and training throughout the provider community. This geographic distribution may also help inform the areas that may benefit most from targeted interventions, such as California, New York, and Texas, which all have large transgender populations.

The data provide particularly valuable insight regarding the burden of chronic conditions in the community, given the incredibly high prevalence of disability and the very high rates of certain conditions. For example, nearly $80 \%$ of the transgender community has been diagnosed with depression during their lifetime. Not only does this signal the heightened level of medical need within the community but it also lays the foundation for future research that examines the prevalence and causes of chronic conditions in the transgender community. Future research could compare the chronic condition burden to the burden found in a matched cohort, helping to clarify the role that being transgender plays in affecting health outcomes. By identifying patterns of transgender health disparities, ranging from discrimination and stigma to the potential long-term effects of hormone therapy, health services researchers will be better able to address the care of transgender persons in the medical setting.

\section{Limitations of Medicare's transgender-related data}

Although CMS's administrative data contain numerous methods for identifying transgender Medicare beneficiaries, these identification methods are not without limitations. CMS data are limited in their ability to identify all transgender beneficiaries because (1) they only identify transgender persons who are medically transitioning and/or have been diagnosed with GID, (2) their administrative data sets contain unobservable error, and (3) billing modifiers, alternative diagnosis codes, and hormone therapy fail to uniquely identify transgender persons.

Because CMS data are based on medical claims for treatment, they only capture persons who are medically transitioning or who have been diagnosed with GID. Focusing on those who are medically transitioning is problematic, given that only $62 \%$ of transgender persons report using hormone therapy. ${ }^{2}$ Although an additional 23\% hope to have hormone therapy in the future, only $62 \%$ to $85 \%$ of transgender persons want or utilize hormone therapy. Therefore, by focusing on medical transitions, this analysis may underestimate the size of the transgender population. Similarly, using ICD-9 codes related to GID may limit the sample, as GID diagnoses are highly controversial in the transgender community, with many transgender persons avoiding the diagnosis. The primary controversy surrounding the diagnosis is that it is considered a mental disorder, which carries the stigma of mental illness and potentially reinforces the gender binary that treats transgender persons as deviant. ${ }^{30}$ Because of this, some transgender persons will avoid the GID diagnosis, requesting other nontransgender-specific diagnoses. Among the most commonly used nontransgenderspecific diagnosis codes is ICD-9 code 259.9 (Unspecified Endocrine Disorder). ${ }^{31,32}$ Because transgender Medicare beneficiaries may not medically transition and/or may actively resist the GID diagnosis, using CMS data to identify transgender Medicare beneficiaries is expected to represent a conservative estimate of Medicare's transgender community.

Errors inherent to administrative data also pose a methodological problem to using administrative data to identify transgender Medicare enrollees. Numerous studies document the limitations of using administrative data to identify diseases, given wide variation in coding accuracy across conditions and settings. ${ }^{33}$ In the Medicare program specifically, a systematic analysis of Medicare claims data compared to medical charts revealed that the percentage of agreement between ICD-9 diagnosis and medical records was, on average, between $73.2 \%$ and $78.2 \%$, with accuracy of diagnosis varying substantially across conditions. ${ }^{34}$ Additional Medicare data validations demonstrate that conditions such as diabetes are highly accurate (100\% claims accuracy), while conditions such as alcohol and drug abuse are highly inaccurate (20\% claims accuracy). ${ }^{35,36}$ Therefore, one can assume that using ICD-9 codes to estimate the transgender Medicare population contains a degree of inherent coding error, which may distort the population estimates.

The final limitation of using CMS's administrative data is the inability of billing modifiers to uniquely identify transgender beneficiaries. While the ICD-9 codes are specific to the transgender community, the billing modifiers are not. Because condition code 45 applies to both the transgender and intersex community, classifying all persons with a condition code 45 modifier as transgender may falsely classify intersex persons as transgender. Intersex persons are different from transgender persons, as they are born with a 
reproductive or sexual anatomy that does not fit typical definitions of male or female, ${ }^{37}$ making them a distinct subgroup of gender minorities. This measurement problem also affects the KX modifier, which applies to multiple types of claims, rather than only those with a gender/procedure conflict. For example, even though the KX modifier might apply to a claim for a transman receiving a female pelvic examination, it might also apply to a female born and identified beneficiary receiving two pelvic examinations in the same calendar year. Because neither of these modifiers applies solely to the transgender community, they cannot be used as a standalone method for classifying beneficiaries as transgender.

Overall, our results demonstrate that administrative data are a valuable resource for identifying the medically transitioning Medicare transgender population and that using ICD-9 codes and billing modifiers are a valid and replicable method that is relevant to many data systems. Using this method, we have made a number of important contributions to the literature, as there are currently no other studies that use Medicare claims data to identify transgender persons. First, we have developed a framework for identifying transgender persons using administrative data, as well as providing a method for validating these results. By replicating the methods outlined in this analysis, researchers can estimate the size of the transgender population and use this data to further analyze health disparities and outcomes in the transgender community. Second, we have provided a baseline description of the diversity and disease burden of the population, laying the foundation for future research programs that expand on this data and statistically model these relationships. Finally, we have proposed numerous avenues of future work to build upon this analysis, including an examination of the intersection between race and gender identity, an examination of the chronic condition burden of transgender persons relative to a matched cohort, and an examination of the underlying causes of chronic conditions in transgender persons. In conclusion, this analysis helps fill the void regarding research on Medicare's transgender population with the goal of informing and encouraging future research on gender minorities.

\section{Disclaimer}

The views expressed in this article are those of the authors and do not necessarily reflect the position or policy of the Centers for Medicare and Medicaid Services, the U.S. Department of Health and Human Services, or NORC at the University of Chicago.

\section{Author Disclosure Statement}

No competing financial interests exist.

\section{References}

1. Graham R, Berkowitz B, Blum R, et al. The Health of Lesbian, Gay, Bisexual, and Transgender People: Building a Foundation for Better Understanding. Washington, DC: Institute of Medicine, 2011.

2. Grant JM, Mottet L, Tanis JE, et al. Injustice at every turn: a report of the national transgender discrimination survey: National Center for Transgender Equality; 2011 [August 18, 2015]. Available from: www .thetaskforce.org/static_html/downloads/reports/reports/ntds_full.pdf (accessed November 22, 2016).

3. Fredriksen-Goldsen $\mathrm{Kl}$, Kim H-J, Emlet $\mathrm{CA}$, et al. The aging and health report: disparities and resilience among lesbian, gay, bisexual, and transgender older adults. Seattle, WA: Institute for Multigenerational Health, 2011.

4. Blosnich JR, Brown GR, Shipherd P, et al. Prevalence of gender identity disorder and suicide risk among transgender veterans utilizing veterans health administration care. Am J Public Health. 2013;103:e27-e32.

5. American Psychological Association Committee on Lesbian G, Bisexual, and Transgender Concerns. Answers to your Questions about Transgender People, Gender Identity, and Gender Expression 2014 [August 18, 2015]. Available from: www.apa.org/topics/lgbt/transgender.aspx (accessed November 22, 2016).

6. Hughes TL, Eliason M. Substance use and abuse in lesbian, gay, bisexual and transgender populations. J Prim Prev. 2002;22:263-298.

7. Jordan KM. Substance abuse among gay, lesbian, bisexual, transgender, and questioning adolescents. School Psychol Rev. 2000;29:201-206.

8. Lombardi EL, van Servellen G. Building culturally sensitive substance use prevention and treatment programs for transgendered populations. J Subst Abuse Treat. 2000;19:291-296.

9. Herbst JH, Jacobs ED, Finlayson TJ, et al. Estimating HIV prevalence and risk behaviors of transgender persons in the United States: a systematic review. AIDS Behav. 2008;12:1-17

10. Clements-Nolle K, Marx R, Katz M. Attempted suicide among transgender persons: the influence of gender-based discrimination and victimization. J Homosex. 2006;51:53-69.

11. Nemoto T, Operario D, Keatley J, Villegas D. Social context of HIV risk behaviours among male-to-female transgenders of colour. AIDS Care. 2004;16:724-735.

12. Mustanski BS, Garofalo R, Emerson EM. Mental health disorders, psychological distress, and suicidality in a diverse sample of lesbian, gay, bisexual, and transgender youths. Am J Public Health. 2010;100:2426-2432.

13. Rotondi NK, Bauer GR, Scanlon K, et al. Prevalence of and risk and protective factors for depression in female-to-male transgender Ontarians: trans PULSE Project. Can J Commun Ment Health. 2012;30:135-155.

14. Centers for Medicare \& Medicaid Services. ICD-9 Code Lookup 2015 [August 18, 2015]. Available from: https://www.cms.gov/Medicare/ Coding/ICD9ProviderDiagnosticCodes/codes.html (accessed November 22, 2016).

15. Centers for Medicare \& Medicaid Services-CMS Manual System. Pub 100-04: Transmittal 1877-Instructions Regarding Processing Claims Rejecting for Gender/Procedure Conflict 2009a [August 18, 2015]. Available from: www.cms.gov/Regulations-and-Guidance/Guidance/ Transmittals/downloads/R1877CP.pdf (accessed November 22, 2016).

16. Centers for Medicare \& Medicaid Services-MLM Matters. MM6638: Instructions Regarding Processing Claims Rejecting for Gender/ Procedure Conflict 2009b. Available from: www.cms.gov/Outreach-andEducation/Medicare-Learning-Network-MLN/MLNMattersArticles/ downloads/MM6638.pdf (accessed November 22, 2016).

17. Arday SL, Arday DR, Monroe S, Zhang J. HCFA's racial and ethnic data: current accuracy and recent improvements. Health Care Financ Rev. 2000;21:107-116.

18. Waldo DR. Accuracy and bias of race/ethnicity codes in the Medicare enrollment database. Health Care Financ Rev. 2004;26:61-72. 
19. Zaslavsky AM, Ayanian JZ, Zaborski LB. The validity of race and ethnicity in enrollment data for Medicare beneficiaries. Health Serv Res. 2012;47(3 pt 2):1300-1321.

20. Boehmer U. Twenty years of public health research: inclusion of lesbian, gay, bisexual, and transgender populations. Am J Public Health. 2002;92:1125-1130.

21. Gornick ME, Eggers PW, Reilly TW, et al. Effects of race and income on mortality and use of services among Medicare beneficiaries. N Engl J Med. 1996;335:791-799.

22. Smedley BD, Stith AY, Nelson AR. Unequal Treatment: Confronting Racial and Ethnic Disparities in Health Care. Washington, DC: National Academies Press, 2002.

23. Virnig $B A$, Lurie $N$, Huang $Z$, et al. Racial variation in quality of care among Medicare + Choice enrollees. Health Aff. 2002;21:224-230.

24. Weech-Maldonado R, Morales LS, Elliott M, et al. Race/ethnicity, language, and patients' assessments of care in Medicaid managed care. Health Serv Res. 2003;38:789-808.

25. Sequist TD, Schneider EC. Addressing racial and ethnic disparities in health care: using federal data to support local programs to eliminate disparities. Health Serv Res. 2006;41(4 pt 1):1451-1468.

26. Schneider KM, O'Donnell BE, Dean D. Prevalence of multiple chronic conditions in the United States' Medicare population. Health Qual Life Outcomes. 2009;7:82.

27. Freid VM, Bernstein AB, Bush MA. Multiple chronic conditions among adults aged 45 and over: trends over the past 10 years. Women. 2012;45:64.

28. Shipherd JC, Mizock L, Maguen S, Green KE. Male-to-female transgender veterans and VA health care utilization. Int J Sex Health. 2012;24:78-87.

29. Knochel KA, Croghan CF, Moone RP, Quam JK. Training, geography, and provision of aging services to lesbian, gay, bisexual, and transgender older adults. J Gerontol Soc Work. 2012;55:426-443.

30. Lev Al. Disordering gender identity: gender identity disorder in the DSMIV-TR. J Psychol Human Sex. 2006;17:35-69.

31. Mayer G. Providing Cross-Gender Hormone Therapy for Transgender Patients Boston, MA: The Fenway Institute, 2013 [August 18, 2015]. Available from: www.lgbthealtheducation.org/wp-content/uploads/ Providing-Cross-Gender-Hormone-Therapy-to-Transgender-Patients.pdf (accessed November 22, 2016).

32. Callen-Lorde Community Health Center. Protocols for the Provision of Cross Gender Hormone Therapy 2012 [August 18, 2015]. Available from: www.tmeltzer.com/assets/callen-lorde-revised-protocols.pdf (accessed November 22, 2016).

33. Yasmeen S, Romano PS, Schembri ME, et al. Accuracy of obstetric diagnoses and procedures in hospital discharge data. Am J Obstet Gynecol. 2006;194:992-1001.

34. Fisher ES, Whaley FS, Krushat WM, et al. The accuracy of Medicare's hospital claims data: progress has been made, but problems remain. Am J Public Health. 1992;82:243-248.

35. Fowles JB, Fowler EJ, Craft C. Validation of claims diagnoses and selfreported conditions compared with medical records for selected chronic diseases. J Ambul Care Manage. 1998;21:24-34.

36. Fowles JB, Lawthers AG, Weiner JP, Garnick DW. Agreement between physicians' office records and Medicare Part B claims data. Health Care Financ Rev. 1995;16:189-199.

37. Intersex Society of North America. What is Intersex? 2015 [August 18, 2015]. Available from: www.isna.org/faq/what_is_intersex (accessed November 22, 2016).

Cite this article as: Proctor K, Haffer SC, Ewald E, Hodge C, James CV (2016) Identifying the transgender population in the Medicare program, Transgender Health 1:1, 250-265, DOI: 10.1089/trgh.2016.0031.

Abbreviations Used
$\mathrm{AIANs}=$ American Indians/Alaska Natives
$\mathrm{API}=$ Asians/Pacific Islanders
$\mathrm{CCW}=$ Chronic Conditions Data Warehouse
$\mathrm{CMS}=$ Centers for Medicare \& Medicaid Services
$\mathrm{FFS}=$ Fee-For-Service
$\mathrm{GID}=$ Gender Identity Disorder
$\mathrm{HCPCS}=$ Healthcare Common Procedure Coding System
$\mathrm{ICD}-9=$ International Classification of Diseases ninth edition
$\mathrm{LGBT}=$ lesbian, gay, bisexual, and transgender
$\mathrm{OASI}=$ Old Age and Survivors Insurance

Appendix Table 1. Gender-Specific Procedure Codes Related to Condition Code 45 and the KX Modifier

\begin{tabular}{|c|c|c|c|c|c|}
\hline HCPCS & Valid sex & Code description & HCPCS & Valid sex & Code description \\
\hline 0071T & Female & U/s leiomyomata ablate $<200$ & 57545 & Female & Remove cervix/repair pelvis \\
\hline 0072T & Female & U/s leiomyomata ablate $>200$ & 57550 & Female & Removal of residual cervix \\
\hline 00842 & Female & Anesth amniocentesis & 57555 & Female & Remove cervix/repair vagina \\
\hline 00846 & Female & Anesth hysterectomy & 57556 & Female & Remove cervix/repair bowel \\
\hline 00851 & Female & Anesth tubal ligation & 57558 & Female & D and $\mathrm{c}$ of cervical stump \\
\hline 00865 & Male & Anesth removal of prostate & 57700 & Female & Revision of cervix \\
\hline 00906 & Female & Anesth removal of vulva & 57720 & Female & Revision of cervix \\
\hline 00908 & Male & Anesth removal of prostate & 57800 & Female & Dilation of cervical canal \\
\hline 00914 & Male & Anesth removal of prostate & 58100 & Female & Biopsy of uterus lining \\
\hline 00920 & Male & Anesth genitalia surgery & 58110 & Female & Bx done w/colposcopy add-on \\
\hline 00921 & Male & Anesth vasectomy & 58120 & Female & Dilation and curettage \\
\hline 00922 & Male & Anesth sperm duct surgery & 58140 & Female & Myomectomy abdominal method \\
\hline 00924 & Male & Anesth testis exploration & 58145 & Female & Myomectomy vaginal method \\
\hline 00926 & Male & Anesth removal of testis & 58146 & Female & Myomectomy abdominal complex \\
\hline 00928 & Male & Anesth removal of testis & 58150 & Female & Total hysterectomy \\
\hline 00930 & Male & Anesth testis suspension & 58152 & Female & Total hysterectomy \\
\hline 00932 & Male & Anesth amputation of penis & 58180 & Female & Partial hysterectomy \\
\hline 00934 & Male & Anesth penis nodes removal & 58200 & Female & Extensive hysterectomy \\
\hline 00936 & Male & Anesth penis nodes removal & 58210 & Female & Extensive hysterectomy \\
\hline 00938 & Male & Anesth insert penis device & 58240 & Female & Removal of pelvis contents \\
\hline 00940 & Female & Anesth vaginal procedures & 58260 & Female & Vaginal hysterectomy \\
\hline 00942 & Female & Anesth surgery on vaginal/urethral & 58262 & Female & Vaginal hysterectomy including t/o \\
\hline
\end{tabular}


Appendix Table 1. (Continued)

\begin{tabular}{|c|c|c|c|c|c|}
\hline HCPCS & Valid sex & Code description & HCPCS & Valid sex & Code description \\
\hline 00944 & Female & Anesth vaginal hysterectomy & 58263 & Female & Vaginal hysterectomy $\mathrm{w} / \mathrm{t} / \mathrm{o}$ and vaginal repair \\
\hline 00948 & Female & Anesth repair of cervix & 58267 & Female & Vaginal hysterectomy w/urinary repair \\
\hline 00950 & Female & Anesth vaginal endoscopy & 58270 & Female & Vaginal hysterectomy w/enterocele repair \\
\hline 00952 & Female & Anesth hysteroscope/graph & 58275 & Female & Hysterectomy/revise vagina \\
\hline 01960 & Female & Anesth vaginal delivery & 58280 & Female & Hysterectomy/revise vagina \\
\hline 01961 & Female & Anesth cs delivery & 58285 & Female & Extensive hysterectomy \\
\hline 01962 & Female & Anesth emergency hysterectomy & 58290 & Female & Vaginal hysterectomy complex \\
\hline 01963 & Female & Anesth cs hysterectomy & 58291 & Female & Vaginal hysterectomy including t/o complex \\
\hline 01965 & Female & Anesth inc/missed ab procedure & 58292 & Female & Vaginal hysterectomy t/o and repair complex \\
\hline 01966 & Female & Anesth induced ab procedure & 58293 & Female & Vaginal hysterectomy w/uro repair complex \\
\hline 01967 & Female & Anesth/analg vaginal delivery & 58294 & Female & Vaginal hysterectomy w/enterocele complex \\
\hline 01968 & Female & Anes/analg cs deliver add-on & 58300 & Female & Insert intrauterine device \\
\hline 01969 & Female & Anesth/analg cs hysterectomy add-on & 58301 & Female & Remove intrauterine device \\
\hline 0336T & Female & Lap ablat uterine fibroids & 58321 & Female & Artificial insemination \\
\hline 0500F & Female & Initial prenatal care visit & 58322 & Female & Artificial insemination \\
\hline 0501F & Female & Prenatal flow sheet & 58323 & Female & Sperm washing \\
\hline $0502 \mathrm{~F}$ & Female & Subsequent prenatal care & 58340 & Female & Catheter for hysterography \\
\hline 0503F & Female & Postpartum care visit & 58345 & Female & Reopen fallopian tube \\
\hline 11976 & Female & Remove contraceptive capsule & 58346 & Female & Insert heyman uteri capsule \\
\hline 19300 & Male & Removal of breast tissue & 58350 & Female & Reopen fallopian tube \\
\hline $3015 F$ & Female & Cerv cancer screen docd & 58353 & Female & Endometrial ablate thermal \\
\hline 36460 & Female & Transfusion service fetal & 58356 & Female & Endometrial cryoablation \\
\hline 37788 & Male & Revascularization penis & 58400 & Female & Suspension of uterus \\
\hline 46744 & Female & Repair of cloacal anomaly & 58410 & Female & Suspension of uterus \\
\hline 46746 & Female & Repair of cloacal anomaly & 58520 & Female & Repair of ruptured uterus \\
\hline 46748 & Female & Repair of cloacal anomaly & 58540 & Female & Revision of uterus \\
\hline 50722 & Female & Release of ureter & 58541 & Female & Lsh uterus $250 \mathrm{~g}$ or less \\
\hline 51845 & Female & Repair bladder neck & 58542 & Female & Lsh w/t/o ut $250 \mathrm{~g}$ or less \\
\hline 51920 & Female & Close bladder-uterus fistula & 58544 & Female & Lsh w/t/o uterus above $250 \mathrm{~g}$ \\
\hline 51925 & Female & Hysterectomy/bladder repair & 58545 & Female & Laparoscopic myomectomy \\
\hline 52010 & Male & Cystoscopy and duct catheter & 58546 & Female & Laparomyomectomy complex \\
\hline 52270 & Female & Cystoscopy and revise urethra & 58548 & Female & Lap radical hyst \\
\hline 52275 & Male & Cystoscopy and revise urethra & 58550 & Female & Laparo-asst vaginal hysterectomy \\
\hline 52285 & Female & Cystoscopy and treatment & 58552 & Female & Laparovaginal hysterectomy including t/o \\
\hline 52402 & Male & Cystourethro cut ejaculatory duct & 58553 & Female & Laparovaginal hysterectomy complex \\
\hline 52450 & Male & Incision of prostate & 58554 & Female & Laparovaginal hysterectomy w/t/o complex \\
\hline 52601 & Male & Prostatectomy (turp) & 58555 & Female & Hysteroscopy dx sep procedure \\
\hline 52647 & Male & Laser surgery of prostate & 58558 & Female & Hysteroscopy biopsy \\
\hline 52648 & Male & Laser surgery of prostate & 58559 & Female & Hysteroscopy lysis \\
\hline 52649 & Male & Prostate laser enucleation & 58560 & Female & Hysteroscopy resect septum \\
\hline 52700 & Male & Drainage of prostate abscess & 58561 & Female & Hysteroscopy remove myoma \\
\hline 53210 & Female & Removal of urethra & 58562 & Female & Hysteroscopy remove fb \\
\hline 53215 & Male & Removal of urethra & 58563 & Female & Hysteroscopy ablation \\
\hline 53230 & Female & Removal of urethra lesion & 58565 & Female & Hysteroscopy sterilization \\
\hline 53235 & Male & Removal of urethra lesion & 58570 & Female & Tlh uterus $250 \mathrm{~g}$ or less \\
\hline 53410 & Male & Reconstruction of urethra & 58571 & Female & Tlh w/t/o $250 \mathrm{~g}$ or less \\
\hline 53415 & Male & Reconstruction of urethra & 58572 & Female & Tlh uterus over $250 \mathrm{~g}$ \\
\hline 53420 & Male & Reconstruct urethra stage 1 & 58573 & Female & Tlh w/t/o uterus over $250 \mathrm{~g}$ \\
\hline 53425 & Male & Reconstruct urethra stage 2 & 58578 & Female & Laparo proc uterus \\
\hline 53430 & Female & Reconstruction of urethra & 58579 & Female & Hysteroscope procedure \\
\hline 53440 & Male & Male sling procedure & 58600 & Female & Division of fallopian tube \\
\hline 53442 & Male & Remove/revise male sling & 58605 & Female & Division of fallopian tube \\
\hline 53502 & Female & Repair of urethra injury & 58611 & Female & Ligate oviduct(s) add-on \\
\hline 53505 & Male & Repair of urethra injury & 58615 & Female & Occlude fallopian tube(s) \\
\hline 53510 & Male & Repair of urethra injury & 58660 & Female & Laparoscopy lysis \\
\hline 53515 & Male & Repair of urethra injury & 58661 & Female & Laparoscopy remove adnexa \\
\hline 53520 & Male & Repair of urethra defect & 58662 & Female & Laparoscopy excise lesions \\
\hline 53600 & Male & Dilate urethra stricture & 58670 & Female & Laparoscopy tubal cautery \\
\hline 53601 & Male & Dilate urethra stricture & 58671 & Female & Laparoscopy tubal block \\
\hline 53605 & Male & Dilate urethra stricture & 58672 & Female & Laparoscopy fimbrioplasty \\
\hline 53620 & Male & Dilate urethra stricture & 58673 & Female & Laparoscopy salpingostomy \\
\hline 53621 & Male & Dilate urethra stricture & 58679 & Female & Laparoscopy procedure oviduct-ovary \\
\hline 53660 & Female & Dilation of urethra & 58700 & Female & Removal of fallopian tube \\
\hline 53661 & Female & Dilation of urethra & 58720 & Female & Removal of ovary/tube(s) \\
\hline 53665 & Female & Dilation of urethra & 58740 & Female & Adhesiolysis tube ovary \\
\hline
\end{tabular}


Appendix Table 1. (Continued)

\begin{tabular}{|c|c|c|c|c|c|}
\hline HCPCS & Valid sex & Code description & HCPCS & Valid sex & Code description \\
\hline 53850 & Male & Prostatic microwave thermotx & 58750 & Female & Repair oviduct \\
\hline 53852 & Male & Prostatic rf thermotx & 58752 & Female & Revise ovarian tube(s) \\
\hline 53855 & Male & Insert prost urethral stent & 58760 & Female & Fimbrioplasty \\
\hline 53860 & Female & Transurethral rf treatment & 58770 & Female & Create new tubal opening \\
\hline 54000 & Male & Slitting of prepuce & 58800 & Female & Drainage of ovarian cyst(s) \\
\hline 54001 & Male & Slitting of prepuce & 58805 & Female & Drainage of ovarian cyst(s) \\
\hline 54015 & Male & Drain penis lesion & 58820 & Female & Drain ovary abscess open \\
\hline 54050 & Male & Destruction penis lesion(s) & 58822 & Female & Drain ovary abscess percut \\
\hline 54055 & Male & Destruction penis lesion(s) & 58825 & Female & Transposition ovary(s) \\
\hline 54056 & Male & Cryosurgery penis lesion(s) & 58900 & Female & Biopsy of ovary(s) \\
\hline 54057 & Male & Laser surgery penis lesion(s) & 58920 & Female & Partial removal of ovary(s) \\
\hline 54060 & Male & Excision of penis lesion(s) & 58925 & Female & Removal of ovarian cyst(s) \\
\hline 54065 & Male & Destruction penis lesion(s) & 58940 & Female & Removal of ovary(s) \\
\hline 54100 & Male & Biopsy of penis & 58943 & Female & Removal of ovary(s) \\
\hline 54110 & Male & Treatment of penis lesion & 58951 & Female & Resect ovarian malignancy \\
\hline 54111 & Male & Treat penis lesion graft & 58952 & Female & Resect ovarian malignancy \\
\hline 54112 & Male & Treat penis lesion graft & 58953 & Female & Tah rad dissect for debulk \\
\hline 54115 & Male & Treatment of penis lesion & 58954 & Female & Tah rad debulk/lymph remove \\
\hline 54120 & Male & Partial removal of penis & 58956 & Female & Bso omentectomy $\mathrm{w} / \mathrm{tah}$ \\
\hline 54125 & Male & Removal of penis & 58957 & Female & Resect recurrent gyn mal \\
\hline 54130 & Male & Remove penis and nodes & 58958 & Female & Resect recur gyn mal w/lym \\
\hline 54135 & Male & Remove penis and nodes & 58960 & Female & Exploration of abdomen \\
\hline 54150 & Male & Circumcision $\mathrm{w} /$ regionl block & 58970 & Female & Retrieval of oocyte \\
\hline 54160 & Male & Circumcision neonate & 58974 & Female & Transfer of embryo \\
\hline 54161 & Male & Circum 28 days or older & 58976 & Female & Transfer of embryo \\
\hline 54162 & Male & Lysis penil circumic lesion & 58999 & Female & Genital surgery procedure \\
\hline 54163 & Male & Repair of circumcision & 59000 & Female & Amniocentesis diagnostic \\
\hline 54164 & Male & Frenulotomy of penis & 59001 & Female & Amniocentesis therapeutic \\
\hline 54200 & Male & Treatment of penis lesion & 59012 & Female & Fetal cord puncture prenatal \\
\hline 54205 & Male & Treatment of penis lesion & 59015 & Female & Chorion biopsy \\
\hline 54220 & Male & Treatment of penis lesion & 59020 & Female & Fetal contract stress test \\
\hline 54230 & Male & Prepare penis study & 59025 & Female & Fetal nonstress test \\
\hline 54231 & Male & Dynamic cavernosometry & 59030 & Female & Fetal scalp blood sample \\
\hline 54235 & Male & Penile injection & 59050 & Female & Fetal monitor w/report \\
\hline 54240 & Male & Penis study & 59051 & Female & Fetal monitor/interpret only \\
\hline 54250 & Male & Penis study & 59070 & Female & Transabdom amnioinfus w/us \\
\hline 54300 & Male & Revision of penis & 59072 & Female & Umbilical cord occlusion w/us \\
\hline 54304 & Male & Revision of penis & 59074 & Female & Fetal fluid drainage $\mathrm{w} / \mathrm{us}$ \\
\hline 54308 & Male & Reconstruction of urethra & 59076 & Female & Fetal shunt placement w/us \\
\hline 54312 & Male & Reconstruction of urethra & 59100 & Female & Remove uterus lesion \\
\hline 54316 & Male & Reconstruction of urethra & 59120 & Female & Treat ectopic pregnancy \\
\hline 54318 & Male & Reconstruction of urethra & 59121 & Female & Treat ectopic pregnancy \\
\hline 54322 & Male & Reconstruction of urethra & 59130 & Female & Treat ectopic pregnancy \\
\hline 54324 & Male & Reconstruction of urethra & 59135 & Female & Treat ectopic pregnancy \\
\hline 54326 & Male & Reconstruction of urethra & 59136 & Female & Treat ectopic pregnancy \\
\hline 54328 & Male & Revise penis/urethra & 59140 & Female & Treat ectopic pregnancy \\
\hline 54332 & Male & Revise penis/urethra & 59150 & Female & Treat ectopic pregnancy \\
\hline 54336 & Male & Revise penis/urethra & 59151 & Female & Treat ectopic pregnancy \\
\hline 54340 & Male & Secondary urethral surgery & 59160 & Female & $\mathrm{D}$ and $\mathrm{c}$ after delivery \\
\hline 54344 & Male & Secondary urethral surgery & 59200 & Female & Insert cervical dilator \\
\hline 54348 & Male & Secondary urethral surgery & 59300 & Female & Episiotomy or vaginal repair \\
\hline 54352 & Male & Reconstruct urethra/penis & 59320 & Female & Revision of cervix \\
\hline 54360 & Male & Penis plastic surgery & 59325 & Female & Revision of cervix \\
\hline 54380 & Male & Repair penis & 59350 & Female & Repair of uterus \\
\hline 54385 & Male & Repair penis & 59400 & Female & Obstetrical care \\
\hline 54390 & Male & Repair penis and bladder & 59409 & Female & Obstetrical care \\
\hline 54400 & Male & Insert semirigid prosthesis & 59410 & Female & Obstetrical care \\
\hline 54401 & Male & Insert self-contd prosthesis & 59412 & Female & Antepartum manipulation \\
\hline 54405 & Male & Insert multi-comp penis prosthesis & 59414 & Female & Deliver placenta \\
\hline 54406 & Male & Remove muti-comp penis pros & 59425 & Female & Antepartum care only \\
\hline 54408 & Male & Repair multi-comp penis prosthesis & 59426 & Female & Antepartum care only \\
\hline 54410 & Male & Remove/replace penis prosthesis & 59430 & Female & Care after delivery \\
\hline 54411 & Male & Remov/replc penis pros comp & 59510 & Female & Cesarean delivery \\
\hline 54415 & Male & Remove self-contd penis pros & 59514 & Female & Cesarean delivery only \\
\hline 54416 & Male & Remv/repl penis contain pros & 59515 & Female & Cesarean delivery \\
\hline
\end{tabular}


Appendix Table 1. (Continued)

\begin{tabular}{|c|c|c|c|c|c|}
\hline HCPCS & Valid sex & Code description & HCPCS & Valid sex & Code description \\
\hline 54417 & Male & Remv/replc penis pros compl & 59525 & Female & Remove uterus after cesarean \\
\hline 54420 & Male & Revision of penis & 59610 & Female & Vbac delivery \\
\hline 54430 & Male & Revision of penis & 59612 & Female & Vbac delivery only \\
\hline 54435 & Male & Revision of penis & 59614 & Female & Vbac care after delivery \\
\hline 54440 & Male & Repair of penis & 59618 & Female & Attempted vbac delivery \\
\hline 54450 & Male & Preputial stretching & 59620 & Female & Attempted vbac delivery only \\
\hline 54500 & Male & Biopsy of testis & 59622 & Female & Attempted vbac after care \\
\hline 54505 & Male & Biopsy of testis & 59812 & Female & Treatment of miscarriage \\
\hline 54512 & Male & Excise lesion testis & 59820 & Female & Care of miscarriage \\
\hline 54520 & Male & Removal of testis & 59821 & Female & Treatment of miscarriage \\
\hline 54522 & Male & Orchiectomy partial & 59830 & Female & Treat uterus infection \\
\hline 54530 & Male & Removal of testis & 59840 & Female & Abortion \\
\hline 54535 & Male & Extensive testis surgery & 59841 & Female & Abortion \\
\hline 54550 & Male & Exploration for testis & 59850 & Female & Abortion \\
\hline 54560 & Male & Exploration for testis & 59851 & Female & Abortion \\
\hline 54600 & Male & Reduce testis torsion & 59852 & Female & Abortion \\
\hline 54620 & Male & Suspension of testis & 59855 & Female & Abortion \\
\hline 54640 & Male & Suspension of testis & 59856 & Female & Abortion \\
\hline 54650 & Male & Orchiopexy (fowler-stephens) & 59857 & Female & Abortion \\
\hline 54660 & Male & Revision of testis & 59866 & Female & Abortion (mpr) \\
\hline 54670 & Male & Repair testis injury & 59870 & Female & Evacuate mole of uterus \\
\hline 54680 & Male & Relocation of testis(es) & 59871 & Female & Remove cerclage suture \\
\hline 54690 & Male & Laparoscopy orchiectomy & 59897 & Female & Fetal invas px w/us \\
\hline 54692 & Male & Laparoscopy orchiopexy & 59898 & Female & Laparo proc ob care/deliver \\
\hline 54699 & Male & Laparoscope proc testis & 59899 & Female & Maternity care procedure \\
\hline 54700 & Male & Drainage of scrotum & 64435 & Female & $\mathrm{N}$ block inj paracervical \\
\hline 54800 & Male & Biopsy of epididymis & 74440 & Male & X-ray male genital tract \\
\hline 54830 & Male & Remove epididymis lesion & 74445 & Male & X-ray examination of penis \\
\hline 54840 & Male & Remove epididymis lesion & 74710 & Female & X-ray measurement of pelvis \\
\hline 54860 & Male & Removal of epididymis & 74740 & Female & X-ray female genital tract \\
\hline 54861 & Male & Removal of epididymis & 74742 & Female & X-ray fallopian tube \\
\hline 54865 & Male & Explore epididymis & 74775 & Female & X-ray examination of perineum \\
\hline 54900 & Male & Fusion of spermatic ducts & 76801 & Female & Ob us $<14$ weeks single fetus \\
\hline 54901 & Male & Fusion of spermatic ducts & 76802 & Female & Ob us $<14$ weeks addl fetus \\
\hline 55000 & Male & Drainage of hydrocele & 76805 & Female & Ob us $>/=14$ weeks sngl fetus \\
\hline 55040 & Male & Removal of hydrocele & 76810 & Female & Ob us $>/=14$ weeks addl fetus \\
\hline 55041 & Male & Removal of hydroceles & 76811 & Female & Ob us detailed sngl fetus \\
\hline 55060 & Male & Repair of hydrocele & 76812 & Female & Ob us detailed addl fetus \\
\hline 55100 & Male & Drainage of scrotum abscess & 76813 & Female & Ob us nuchal meas 1 gest \\
\hline 55110 & Male & Explore scrotum & 76814 & Female & Ob us nuchal meas add-on \\
\hline 55120 & Male & Removal of scrotum lesion & 76815 & Female & Ob us limited fetus(s) \\
\hline 55150 & Male & Removal of scrotum & 76816 & Female & Ob us follow-up per fetus \\
\hline 55175 & Male & Revision of scrotum & 76817 & Female & Transvaginal us obstetric \\
\hline 55180 & Male & Revision of scrotum & 76818 & Female & Fetal biophys profile $w / n s t$ \\
\hline 55200 & Male & Incision of sperm duct & 76819 & Female & Fetal biophys profile w/o nst \\
\hline 55250 & Male & Removal of sperm duct(s) & 76825 & Female & Echo examination of fetal heart \\
\hline 55300 & Male & Prepare sperm duct $x$-ray & 76826 & Female & Echo examination of fetal heart \\
\hline 55400 & Male & Repair of sperm duct & 76827 & Female & Echo examination of fetal heart \\
\hline 55450 & Male & Ligation of sperm duct & 76828 & Female & Echo examination of fetal heart \\
\hline 55500 & Male & Removal of hydrocele & 76830 & Female & Transvaginal us non-ob \\
\hline 55520 & Male & Removal of sperm cord lesion & 76831 & Female & Echo examination uterus \\
\hline 55540 & Male & Revise hernia and sperm veins & 76941 & Female & Echo guide for transfusion \\
\hline 55550 & Male & Laparo ligate spermatic vein & 76945 & Female & Echo guide villus sampling \\
\hline 55559 & Male & Laparo proc spermatic cord & 76946 & Female & Echo guide for amniocentesis \\
\hline 55600 & Male & Incise sperm duct pouch & 76948 & Female & Echo guide ova aspiration \\
\hline 55605 & Male & Incise sperm duct pouch & 77057 & Female & Mammogram screening \\
\hline 55650 & Male & Remove sperm duct pouch & 78761 & Male & Testicular imaging w/flow \\
\hline 55680 & Male & Remove sperm pouch lesion & 80055 & Female & Obstetric panel \\
\hline 55700 & Male & Biopsy of prostate & 81025 & Female & Urine pregnancy test \\
\hline 55705 & Male & Biopsy of prostate & 81500 & Female & Onco (ovar) two proteins \\
\hline 55706 & Male & Prostate saturation sampling & 81503 & Female & Onco (ovar) five proteins \\
\hline 55720 & Male & Drainage of prostate abscess & 81507 & Female & Fetal aneuploidy trisom risk \\
\hline 55725 & Male & Drainage of prostate abscess & 81508 & Female & Ftl cgen abnor two proteins \\
\hline 55801 & Male & Removal of prostate & 81509 & Female & Ftl cgen abnor three proteins \\
\hline
\end{tabular}


Appendix Table 1. (Continued)

\begin{tabular}{|c|c|c|c|c|c|}
\hline HCPCS & Valid sex & Code description & HCPCS & Valid sex & Code description \\
\hline 55810 & Male & Extensive prostate surgery & 81510 & Female & Ftl cgen abnor three anal \\
\hline 55812 & Male & Extensive prostate surgery & 81511 & Female & Ftl cgen abnor four anal \\
\hline 55815 & Male & Extensive prostate surgery & 81512 & Female & Ftl cgen abnor five anal \\
\hline 55821 & Male & Removal of prostate & 82120 & Female & Amines vaginal fluid qual \\
\hline 55831 & Male & Removal of prostate & 82143 & Female & Amniotic fluid scan \\
\hline 55840 & Male & Extensive prostate surgery & 82731 & Female & Assay of fetal fibronectin \\
\hline 55842 & Male & Extensive prostate surgery & 84112 & Female & Eval amniotic fluid protein \\
\hline 55845 & Male & Extensive prostate surgery & 84135 & Female & Assay of pregnanediol \\
\hline 55860 & Male & Surgical exposure prostate & 84138 & Female & Assay of pregnanetriol \\
\hline 55862 & Male & Extensive prostate surgery & 84152 & Male & Assay of psa complexed \\
\hline 55865 & Male & Extensive prostate surgery & 84153 & Male & Assay of psa total \\
\hline 55866 & Male & Laparo radical prostatectomy & 84154 & Male & Assay of psa free \\
\hline 55870 & Male & Electroejaculation & 84163 & Female & Pappa serum \\
\hline 55873 & Male & Cryoablate prostate & 84830 & Female & Ovulation tests \\
\hline 55875 & Male & Transperi needle place pros & 85460 & Female & Hemoglobin fetal \\
\hline 55876 & Male & Place rt device/marker pros & 85461 & Female & Hemoglobin fetal \\
\hline 55899 & Male & Genital surgery procedure & 88141 & Female & Cytopath c/v interpret \\
\hline 55970 & Male & Sex transformation $m$ to $f$ & 88142 & Female & Cytopath $c / v$ thin layer \\
\hline 55980 & Female & Sex transformation $\mathrm{f}$ to $\mathrm{m}$ & 88143 & Female & Cytopath $\mathrm{c} / \mathrm{v}$ thin layer redo \\
\hline 56405 & Female & $\mathrm{I}$ and $\mathrm{d}$ of vulva/perineum & 88147 & Female & Cytopath $\mathrm{c} / \mathrm{v}$ automated \\
\hline 56420 & Female & Drainage of gland abscess & 88148 & Female & Cytopath $\mathrm{c} / \mathrm{v}$ auto rescreen \\
\hline 56440 & Female & Surgery for vulva lesion & 88150 & Female & Cytopath $\mathrm{c} / \mathrm{v}$ manual \\
\hline 56441 & Female & Lysis of labial lesion(s) & 88153 & Female & Cytopath $\mathrm{c} / \mathrm{v}$ redo \\
\hline 56442 & Female & Hymenotomy & 88154 & Female & Cytopath $\mathrm{c} / \mathrm{v}$ select \\
\hline 56501 & Female & Destroy vulva lesions sim & 88155 & Female & Cytopath $c / v$ index add-on \\
\hline 56515 & Female & Destroy vulva lesion/s compl & 88164 & Female & Cytopath tbs c/v manual \\
\hline 56605 & Female & Biopsy of vulva/perineum & 88165 & Female & Cytopath tbs c/v redo \\
\hline 56606 & Female & Biopsy of vulva/perineum & 88166 & Female & Cytopath tbs $\mathrm{c} / \mathrm{v}$ auto redo \\
\hline 56620 & Female & Partial removal of vulva & 88167 & Female & Cytopath tbs $\mathrm{c} / \mathrm{v}$ select \\
\hline 56625 & Female & Complete removal of vulva & 88174 & Female & Cytopath $\mathrm{c} / \mathrm{v}$ auto in fluid \\
\hline 56630 & Female & Extensive vulva surgery & 88175 & Female & Cytopath $\mathrm{c} / \mathrm{v}$ auto fluid redo \\
\hline 56631 & Female & Extensive vulva surgery & 88267 & Female & Chromosome analysis placenta \\
\hline 56632 & Female & Extensive vulva surgery & 88269 & Female & Chromosome analysis amniotic \\
\hline 56633 & Female & Extensive vulva surgery & 89264 & Male & Identify sperm tissue \\
\hline 56634 & Female & Extensive vulva surgery & 89300 & Female & Semen analysis w/huhner \\
\hline 56637 & Female & Extensive vulva surgery & 89310 & Male & Semen analysis w/count \\
\hline 56640 & Female & Extensive vulva surgery & 89320 & Male & Semen anal vol/count/mot \\
\hline 56700 & Female & Partial removal of hymen & 89321 & Male & Semen anal sperm detection \\
\hline 56805 & Female & Repair clitoris & 89329 & Male & Sperm evaluation test \\
\hline 56810 & Female & Repair of perineum & 89330 & Male & Evaluation cervical mucus \\
\hline 56820 & Female & Examination of vulva w/scope & 89331 & Male & Retrograde ejaculation anal \\
\hline 56821 & Female & Examination/biopsy of vulva w/scope & 99500 & Female & Home visit prenatal \\
\hline 57000 & Female & Exploration of vagina & 99501 & Female & Home visit postnatal \\
\hline 57010 & Female & Drainage of pelvic abscess & A4261 & Female & Cervical cap contraceptive \\
\hline 57020 & Female & Drainage of pelvic fluid & A4264 & Female & Intratubal occlusion device \\
\hline 57022 & Female & I and d vaginal hematoma $\mathrm{pp}$ & A4266 & Female & Diaphragm \\
\hline 57023 & Female & I and d vaginal hematoma non-ob & A4267 & Male & Male condom \\
\hline 57061 & Female & Destroy vaginal lesions simple & A4268 & Female & Female condom \\
\hline 57065 & Female & Destroy vaginal lesions complex & A4269 & Female & Spermicide \\
\hline 57100 & Female & Biopsy of vagina & A4281 & Female & Replacement breast pump tube \\
\hline 57105 & Female & Biopsy of vagina & A4282 & Female & Replacement breast pump adpt \\
\hline 57106 & Female & Remove vagina wall partial & A4283 & Female & Replacement breast pump cap \\
\hline 57107 & Female & Remove vagina tissue part & A4284 & Female & Replacement breast pump shield \\
\hline 57109 & Female & Vaginectomy partial w/nodes & A4285 & Female & Replacement breast pump bottle \\
\hline 57110 & Female & Remove vagina wall complete & A4286 & Female & Replacement breastpump lok ring \\
\hline 57111 & Female & Remove vagina tissue compl & A4326 & Male & Male external catheter \\
\hline 57112 & Female & Vaginectomy $\mathrm{w} /$ nodes compl & A4327 & Female & Female urinary collect dev cup \\
\hline 57120 & Female & Closure of vagina & A4328 & Female & Female urinary collect pouch \\
\hline 57130 & Female & Remove vagina lesion & C9739 & Male & Cystoscopy prostatic imp 1-3 \\
\hline 57135 & Female & Remove vagina lesion & C9740 & Male & Cysto impl 4 or more \\
\hline 57150 & Female & Treat vagina infection & E0325 & Male & Urinal male jug type \\
\hline 57155 & Female & Insert uteri tandem/ovoids & E0326 & Female & Urinal female jug type \\
\hline 57156 & Female & Ins vaginal brachytx device & E0602 & Female & Manual breast pump \\
\hline 57160 & Female & Insert pessary/other device & E0603 & Female & Electric breast pump \\
\hline
\end{tabular}


Appendix Table 1. (Continued)

\begin{tabular}{|c|c|c|c|c|c|}
\hline HCPCS & Valid sex & Code description & HCPCS & Valid sex & Code description \\
\hline 57170 & Female & Fitting of diaphragm/cap & E0604 & Female & Hosp grade elec breast pump \\
\hline 57180 & Female & Treat vaginal bleeding & G0027 & Male & Semen analysis \\
\hline 57200 & Female & Repair of vagina & G0101 & Female & Ca screen; pelvic/breast exam \\
\hline 57210 & Female & Repair vagina/perineum & G0102 & Male & Prostate ca screening; dre \\
\hline 57220 & Female & Revision of urethra & G0103 & Male & Psa screening \\
\hline 57230 & Female & Repair of urethral lesion & G0123 & Female & Screen cerv/vaginal thin layer \\
\hline 57240 & Female & Repair bladder and vagina & G0124 & Female & Screen $\mathrm{c} / \mathrm{v}$ thin layer by md \\
\hline 57250 & Female & Repair rectum and vagina & G0141 & Female & Scr c/v cyto, autosys and md \\
\hline 57260 & Female & Repair of vagina & G0143 & Female & Scr c/v cyto, thin layer, rescr \\
\hline 57265 & Female & Extensive repair of vagina & G0144 & Female & Scr c/v cyto, thin layer, rescr \\
\hline 57267 & Female & Insert mesh/pelvic flr addon & G0145 & Female & Scr c/v cyto, thin layer, rescr \\
\hline 57268 & Female & Repair of bowel bulge & G0147 & Female & Scr c/v cyto, automated sys \\
\hline 57270 & Female & Repair of bowel pouch & G0148 & Female & Scr c/v cyto, autosys, rescr \\
\hline 57280 & Female & Suspension of vagina & G0202 & Female & Screeningmammographydigital \\
\hline 57282 & Female & Colpopexy extraperitoneal & G0416 & Male & Biopsy prostate $10-20$ \\
\hline 57283 & Female & Colpopexy intraperitoneal & G0417 & Male & Biopsy prostate $21-40$ \\
\hline 57284 & Female & Repair paravag defect open & G0418 & Male & Biopsy prostate $41-60$ \\
\hline 57285 & Female & Repair paravag defect vaginal & G0419 & Male & Biopsy prostate: $>60$ \\
\hline 57287 & Female & Revise/remove sling repair & G0458 & Male & Ldr prostate brachy comp rat \\
\hline 57288 & Female & Repair bladder defect & G8806 & Female & Transab or transvag us \\
\hline 57289 & Female & Repair bladder and vagina & G8807 & Female & Doc reas no us \\
\hline 57291 & Female & Construction of vagina & G8808 & Female & No transab or transvag us \\
\hline 57292 & Female & Construct vagina with graft & G8809 & Female & Rh-immunoglobulin order \\
\hline 57295 & Female & Revise vaginal graft through vagina & G8810 & Female & Doc reas no rh-immuno \\
\hline 57296 & Female & Revise vaginal graft open abd & G8811 & Female & No rh-immunoglobulin order \\
\hline 57307 & Female & Fistula repair and colostomy & P3000 & Female & Screen pap by tech w md supv \\
\hline 57308 & Female & Fistula repair transperine & P3001 & Female & Screening pap smear by phys \\
\hline 57310 & Female & Repair urethrovaginal lesion & Q0091 & Female & Obtaining screen pap smear \\
\hline 57311 & Female & Repair urethrovaginal lesion & S0610 & Female & Annual gynecological examina \\
\hline 57320 & Female & Repair bladder-vagina lesion & S0612 & Female & Annual gynecological examina \\
\hline 57330 & Female & Repair bladder-vagina lesion & S4005 & Female & Interim labor facility globa \\
\hline 57335 & Female & Repair vagina & S4011 & Female & IVF package \\
\hline 57400 & Female & Dilation of vagina & S4013 & Female & Complete GIFT case rate \\
\hline 57410 & Female & Pelvic examination & S4014 & Female & Complete ZIFT case rate \\
\hline 57415 & Female & Remove vaginal foreign body & S4015 & Female & Complete IVF nos case rate \\
\hline 57420 & Female & Examination of vagina w/scope & S4016 & Female & Frozen IVF case rate \\
\hline 57421 & Female & Examination/biopsy of vaginal $\mathrm{w} / \mathrm{scope}$ & S4017 & Female & IVF canc a stim case rate \\
\hline 57423 & Female & Repair paravag defect lap & S4018 & Female & F EMB trns canc case rate \\
\hline 57425 & Female & Laparoscopy surg colpopexy & S4020 & Female & IVF canc a aspir case rate \\
\hline 57426 & Female & Revise prosth vaginal graft lap & S4021 & Female & IVF canc $\mathrm{p}$ aspir case rate \\
\hline 57452 & Female & Examination of cervix w/scope & S4022 & Female & Asst oocyte fert case rate \\
\hline 57454 & Female & Bx/curett of cervix w/scope & S4023 & Female & Incomplete donor egg case rate \\
\hline 57455 & Female & Biopsy of cervix w/scope & S4025 & Female & Donor serv IVF case rate \\
\hline 57456 & Female & Endocerv curettage w/scope & S4026 & Male & Procure donor sperm \\
\hline 57460 & Female & Bx of cervix w/scope leep & S4027 & Female & Store prev frozen embryos \\
\hline 57461 & Female & Conz of cervix w/scope leep & S4028 & Male & Microsurg epi sperm asp \\
\hline 57500 & Female & Biopsy of cervix & S4030 & Male & Sperm procure init visit \\
\hline 57505 & Female & Endocervical curettage & S4031 & Male & Sperm procure subs visit \\
\hline 57510 & Female & Cauterization of cervix & S4035 & Female & Stimulated IUI case rate \\
\hline 57511 & Female & Cryocautery of cervix & S4037 & Female & Cryo embryo transf case rate \\
\hline 57513 & Female & Laser surgery of cervix & S4040 & Female & Monit store cryo embryo $30 \mathrm{~d}$ \\
\hline 57520 & Female & Conization of cervix & S4989 & Female & Contracept IUD \\
\hline 57522 & Female & Conization of cervix & S4993 & Female & Contraceptive pills for bc \\
\hline 57530 & Female & Removal of cervix & S9001 & Female & Home uterine monitor with or \\
\hline 57531 & Female & Removal of cervix radical & S9436 & Female & Lamaze class \\
\hline \multirow[t]{3}{*}{57540} & Female & Removal of residual cervix & S9437 & Female & Childbirth refresher class \\
\hline & & & S9438 & Female & Cesarean birth class \\
\hline & & & S9439 & Female & VBAC class \\
\hline
\end{tabular}

HCPCS, Healthcare Common Procedure Coding System. 
Appendix Table 2. Sex Hormones

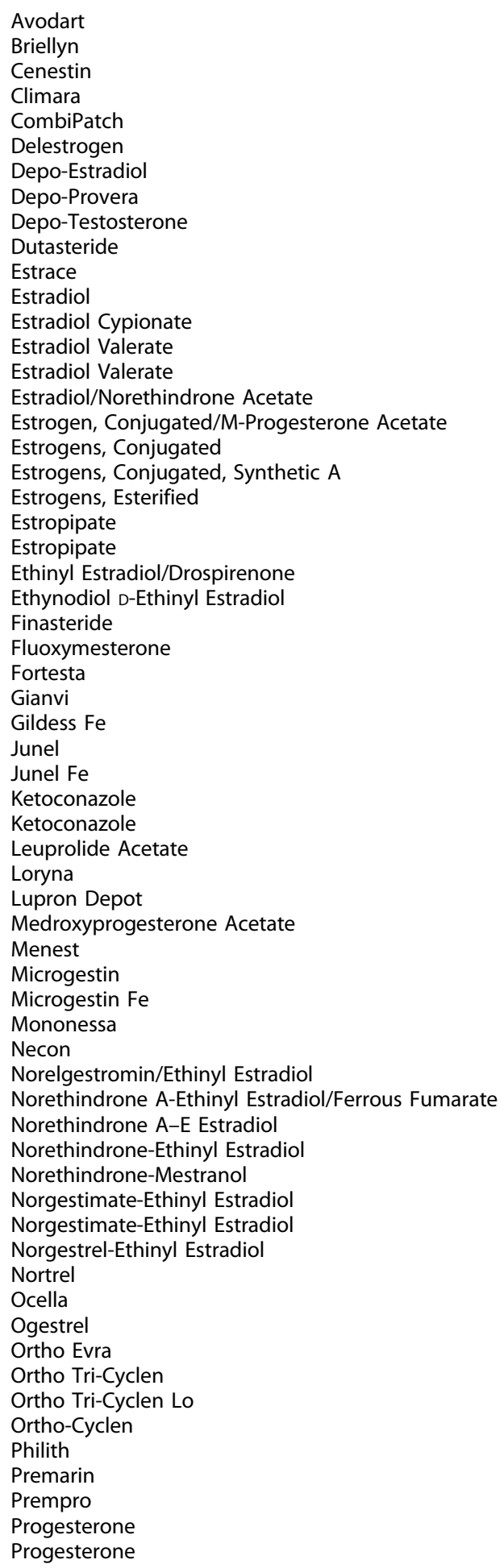

(continued)
Appendix Table 2. (Continued)
Progesterone, Micronized

Spironolactone

Spironolactone

Sprintec

Syeda

Testim

Testosterone

Testosterone Cypionate

Testosterone Cypionate

Testosterone Enanthate

Testosterone Enanthate

Tri-Linyah

TriNessa

Tri-Previfem

Tri-Sprintec

Xolege

Zovia 1-35E

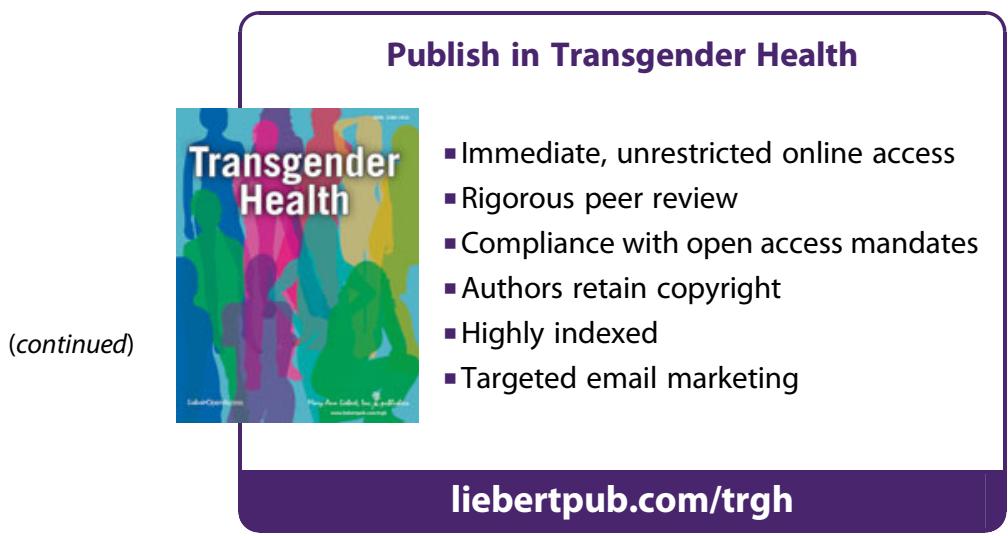

